\title{
Eficiência de inseticidas no controle de Chrysodeixis includens (Lepidoptera: Noctuidae) na cultura da soja
}

\author{
Gustavo Luís Mamoré Martins ${ }^{1}$, Germison Vital Tomquelski² \\ ${ }^{1}$ Universidade Estadual de Mato Grosso do Sul, Unidade Universitária de Cassilândia, Cassilândia, Mato Grosso do Sul, Brasil. \\ E-mail: gustavomamore@yahoo.com.br \\ ${ }^{2}$ Fundação de Apoio a Pesquisa Agropecuária de Chapadão do Sul, Chapadão do Sul, Mato Grosso do Sul, Brasil. \\ E-mail: germison@fundacaochapadao.com.br
}

Recebido: 08/09/2015; Aceito: 28/11/2015.

\section{RESUMO}

O objetivo do trabalho foi estudar a eficiência de alguns inseticidas para o controle de Chrysodeixis includens (Lepidoptera: Noctuidae) em duas safras na cultura da soja. Desenvolveu-se dois experimentos, sendo um na safra 2006/07 (experimento 1) e outro na safra 2007/08 (Experimento 2), no município de Chapadão do Sul (MS), sendo delineados em blocos ao acaso com 7 tratamentos e 4 repetições (experimento 1) e 6 tratamentos e 4 repetições (experimento 2). No experimento 1, os tratamentos foram: 1. Testemunha; 2. Profenofós + Lufenuron (151 g i.a. $\left.\mathrm{ha}^{-1}\right)$; 3. Metomil (7,5 g i.a. ha $\left.{ }^{-1}\right) ; 4$. Teflubenzuron (384 g i.a. ha $\left.{ }^{-1}\right)$; 5. Thiodicarb (24 g i.a. ha $\left.{ }^{-1}\right) ; 6$. Clorpirifós $\left(28,8 \mathrm{~g}\right.$ i.a. $\left.\mathrm{ha}^{-1}\right)$; 7. Fenitrotion + esfenvarelate $\left(384 \mathrm{~g}\right.$ i.a. ha $\left.{ }^{-1}\right)$. No experimento 2, foram utilizados os tratamentos: 1. Testemunha; 2. Teflubenzuron (12 g i.a. ha-1); 3. Metomil (129 g i.a. ha $\left.{ }^{-1}\right) ; 4$. Profenofós + Lufenuron $(15+150$ g i.a. ha $\left.{ }^{-1}\right) ; 5$. Flubendiamid $\left(12 \mathrm{~g}\right.$ i.a. ha $\left.{ }^{-1}\right) ; 6$. Flubendiamid $\left(14,4 \mathrm{~g}\right.$ i.a. ha $\left.{ }^{-1}\right)$. Foi observada uma baixa eficiência dos inseticidas no controle de $C$. includens na cultura da soja. Os inseticidas Fenitrotion+Esfenvarelate, Tiodicarb, Metomil e Clorpirifós são os mais eficientes para o controle de lagartas pequenas de falsa-medideira (Chrysodeixis includens). Para as lagartas grandes os inseticidas mais eficientes são Flubendiamide e Profenofós+Lufenuron.

Palavras-chave: Glycine max, lagarta, controle químico.

\section{Efficiency of insecticides on Chrysodeixis includens (Lepidoptera: Noctuidae) on soybean crop}

\section{ABSTRACT}

The objective of study was to investigated the efficiency of insecticides to control of Chrysodeixis includens (Lepidoptera: Noctuidae) on soybean crop. Two experiments were conducted: the first in 2006/07 and second in 2007/08 in the municipality of Chapadão do Sul, MS, Brazil. An randomized block design with six treatments and four replications (Experiment 1) and seven treatments and four replications (Experiment 2) were used. In experiment 1, the treatments tested were: 1) Control, 2) Profenofos + Lufenuron (151 g i.a. ha $\left.\left.{ }^{-1}\right), 3\right)$ Methomyl (7.5 g i.a. ha $\left.\left.{ }^{-1}\right), 4\right)$ Teflubenzuron (384 g i.a. ha $\left.\left.{ }^{-1}\right), 5\right)$ thiodicarb (24 g i.a. ha-1), 6) Chlorpyrifos $\left.\left(28.8 \mathrm{~g}^{\text {i.a. }} \mathrm{ha}^{-1}\right), 7\right)$ Fenitrothion + Esfenvarelate ( 384 g i.a. ha $\left.{ }^{-1}\right)$. In experiment 2, treatments tested were: 1) Control, 2) Teflubenzuron $\left(12\right.$ g i.a. ha $\left.\left.{ }^{-1}\right), 3\right)$ Methomyl (129 g i.a. ha $\left.{ }^{-1}\right)$, 4) Profenofos + Lufenuron (15+150 g i.a. ha $\left.{ }^{-1}\right)$, 5) Flubendiamid (12 g i.a. ha $\left.{ }^{-1}\right)$, 6) Flubendiamid $\left(14,4\right.$ g i.a. ha $\left.{ }^{-1}\right)$. An low efficiency of insecticides to control of $C$. includens in soybean crop was observed. The insecticides Fenitrothion + Esfenvarelate, Thiodicarb, Methomyl and Chlorpyrifos are the most efficient for the control of small caterpillars of Chrysodeixis includens (Lepidoptera: Noctuidae). For the large caterpillars the most effective insecticides are Flubendiamide and Profenofos + Lufenuron.

Key words: Glycine max, caterpillar, chemical control. 


\section{Introdução}

A cultura da soja apresenta uma grande área cultivada no Brasil, na safra de 2014/15 obteve uma produção de 96,0 milhões de toneladas e produtividade de $3.011 \mathrm{~kg} \mathrm{ha}^{-1}$ (CONAB, 2015). Nessa cultura, um dos principais fatores que afetam a produtividade é a ocorrência de pragas (TOMQUELSKI et al., 2015). A lagarta falsa-medideira - Chrysodeixis includens (Lepidoptera: Noctuidae), até o final da década de 90, era considerada praga secundária, sendo encontrada com maior frequência a partir da safra agrícola de 2003 (EMBRAPA, 2008). Atualmente, os cuidados no controle dessa praga são tomados praticamente durante todo o período de desenvolvimento das plantas, em decorrência da alta capacidade de consumo de área foliar (que pode chegar até $200 \mathrm{~cm}^{2}$ ) e por estar presente em altas populações em praticamente todas as lavouras de soja da região das últimas safras. Além da desfolha, é comum se observar a campo o ataque de lagartas, geralmente grandes, em vagens já formadas de soja, potencializando os danos causados pela praga (TOMQUELSKI et al., 2015).

A espécie $C$. includens pode reduzir drasticamente a área foliar e ocasionar intenso dano econômico, especialmente se a desfolha ocorrer durante o período reprodutivo da cultura. Esta espécie é mais tolerante às dosagens usuais dos inseticidas quando comparadas com a lagarta-da-soja (Anticarsia gemmatalis). Além disso, devido ao hábito que a lagarta falsa-medideira tem de ficar "escondida" mais internamente entre as folhas das plantas, a aplicação de inseticidas deve atingir a praga no "baixeiro" e no interior das plantas de soja cuja consequência direta é a necessidade de utilização de doses elevadas de produtos químicos para melhorar o controle (DEGRANDE; VIVAN, 2008). Outro ponto a considerar no combate a esta praga é a sua ótima adaptação em condições de seca (GALLO et al., 2002).

Para a tomada de decisão no controle da lagarta é necessário realizar amostragem nas lavouras (TOMQUELSKI; MARTINS, 2012). Após verificar um nível populacional capaz de causar danos econômicos, torna-se necessário a utilização de defensivos agrícolas. O uso de inseticidas é uma ferramenta de manejo de pragas (MARTINS et al., 2009; MARTINS et al., 2013; MARTINS et al., 2015). Os inseticidas utilizados no controle de lagartas impedem a ocorrência de dano econômico na cultura da soja (SILVA et al., 2003).

Pesquisas avaliaram a eficiência de inseticidas no controle de $C$. includens na cultura da soja (TOMQUELSKI; MARTINS, 2006) com diferentes porcentagens de eficiências de controle. Os diferentes grupos químicos de inseticidas com seus modos de ação específicos proporcionam distintas eficiências. Os principais grupos químicos de inseticidas aplicados nas lavouras de soja são os piretróides, organofosforados e carbamatos utilizados há várias décadas (TOMQUELSKI et al., 2015). As benzoiluréias e diamida do ácido ftálico têm sido pesquisadas nos últimos anos (GALLO et al., 2002; HANNIG et al., 2009).

Os piretróides e organofosforados se caracterizam por apresentar amplo espectro de ação, custo reduzido, podendo ainda ser utilizados em populações de pragas em estádios mais avançados de desenvolvimento (SOSA-GÓMEZ, 2000). Os carbamatos inibem a ação da enzima acetilcolinesterase e são neurotóxicos para os insetos-pragas (GALLO et al., 2002). As benzoiluréias são os principais representantes dos inseticidas do grupo químico dos inibidores da síntese de quitina com um bom efeito residual no controle de lagartas (SILVA et al., 2003). Os inseticidas do grupo químico diamida do ácido ftálico apresentam eficiência contra diferentes espécies de lagartas e seletividade aos inimigos naturais (HANNIG et al., 2009; GUEDES et al., 2012).

Apesar do grande número de inseticidas disponíveis no mercado, observa-se em lavouras de soja, baixa eficiência dos produtos no controle de $C$. includens em algumas regiões. Deste modo, o objetivo foi avaliar a eficiência de inseticidas sobre $C$. includens na cultura da soja, em condições de campo.

\section{Material e Métodos}

Neste estudo foram conduzidos dois experimentos, no município de Chapadão do Sul (MS), na área experimental da Fundação de Apoio a Pesquisa Agropecuária de Chapadão (Fundação Chapadão).

\section{Experimento 1}

O experimento foi instalado no sistema plantio direto, em uma área que foi semeada com a cultura do milho no ano agrícola 2005/2006, pousio na entre safra e semeadura da cultura na safra de 2006/2007. O cultivar de soja utilizado foi o FMT - Tucunaré, com espaçamento de $0,45 \mathrm{~m}$ entre linhas.

A adubação de semeadura consistiu de $150 \mathrm{~kg} \mathrm{ha}^{-1}$ da formulação 02-20-20 + micronutrientes. O controle das plantas daninhas foi realizado com $50 \mathrm{~g} \mathrm{ha}^{-1} \mathrm{de}$ Clorimuron-ethyl $+500 \mathrm{~mL} \mathrm{ha}^{-1}$ de lactofen aos 21 dias após a emergência. $\mathrm{O}$ controle de doenças foi realizado com aplicação de Azoxistrobina + Ciproconazol 300 $\mathrm{mL} \mathrm{ha} \mathrm{p}^{-1}+$ óleo mineral paranínfico $600 \mathrm{~mL} \mathrm{ha}^{-1}$ num total de três aplicações.

$\mathrm{O}$ delineamento experimental foi blocos ao acaso com sete tratamentos e quatro repetições. Os 
tratamentos com respectivos ingredientes ativos, doses, grupos químicos e modos de ação estão descritos na Tabela 1. Cada parcela foi constituída por 14 linhas de semeadura de $10 \mathrm{~m}$ de comprimento, perfazendo uma área total de $63 \mathrm{~m}^{2}$.

Foi realizada uma aplicação dos inseticidas, aos 66 dias após a emergência (DAE), época em que a cultura se encontrava no estádio reprodutivo R2. Nas aplicações utilizou-se um pulverizador $\mathrm{CO}_{2}$ pressurizado, com barra de 3,0 m e pontas do tipo leque 11002, operando com pressão constante de 35 PSI e volume de calda de $150 \mathrm{~L} \mathrm{ha}^{-1}$. As condições climáticas médias durante a aplicação foram: umidade relativa $80 \%$, temperatura de $23{ }^{\circ} \mathrm{C}$ e velocidade do vento de $3 \mathrm{~km} \mathrm{~h}^{-1}$.

Foram realizadas avaliações nas parcelas, utilizando-se um pano com as dimensões de $1 \mathrm{~m} 2$, com 16 batidas de pano por tratamento, contando-se o número de lagartas grandes $(>1,5 \mathrm{~cm})$ e pequenas $(<$ $1,5 \mathrm{~cm}$ ) aos 0 (Prévia) 2, 4, 7, 14 e 21 dias após a aplicação.

\section{Experimento 2}

O experimento foi instalado no sistema plantio direto. O cultivar de soja utilizado foi o BRS-Valiosa RR, semeado em 03 de dezembro de 2007, com espaçamento de $0,45 \mathrm{~m}$ entre linhas. A emergência das plantas ocorreu no dia 12 de dezembro de 2007.

A adubação de semeadura consistiu de $350 \mathrm{~kg} \mathrm{ha}^{-1}$ da formulação $02-25-10+15 \mathrm{~kg} \mathrm{ha}^{-1}$ de ourofós +100 $\mathrm{kg} \mathrm{ha}^{-1}$ de $\mathrm{KCl}\left(60 \%\right.$ de $\left.\mathrm{K}_{2} \mathrm{O}\right)$ a lanço aos 25 dias após a emergência. $\mathrm{O}$ controle das plantas daninhas foi realizado com clorimuron $50 \mathrm{~g} \mathrm{ha}^{-1}+$ glifosato nortox 2,5 $\mathrm{L} \mathrm{ha}^{-1}$ aos 21 dias após a emergência.

$\mathrm{O}$ delineamento experimental foi blocos ao acaso com seis tratamentos e quatro repetições. Os tratamentos com respectivos ingredientes ativos, doses, grupos químicos e modos de ação estão descritos na Tabela 1.

Cada parcela foi constituída por 14 linhas de semeadura de $10 \mathrm{~m}$ de comprimento, perfazendo uma área total de $63 \mathrm{~m}^{2}$.

Foi realizada uma aplicação, sendo esta com a soja no estádio V8, na data de 30/01/2009. Para as aplicações utilizou-se um pulverizador $\mathrm{CO}_{2}$ pressurizado, com barra de três metros e pontas do tipo leque 11002, operando com pressão constante de 35 PSI e volume de calda de $150 \mathrm{~L} \mathrm{ha}^{-1}$.

As condições climáticas médias durante a aplicação foram: umidade relativa $75 \%$, temperatura de $26,5^{\circ} \mathrm{C}$ e velocidade do vento de $4 \mathrm{~km} \mathrm{~h}^{-1}$.

Foram realizadas avaliações nas parcelas, utilizando-se um pano com as dimensões de $1 \mathrm{~m} 2$, com 04 batidas de pano por parcela, sendo contado o número de lagartas grandes $(>1,5 \mathrm{~cm})$ e pequenas $(<1,5 \mathrm{~cm})$ aos 0 (Prévia) 2, 4, 7 e 14 dias após a aplicação

Para a análise de dados utilizou-se o programa SAEG - Sistemas para Análises Estatísticas e Genéticas (UFV, 2001). Os dados foram submetidos à análise de variância e as médias comparadas pelo teste Tukey a 5 $\%$ de probabilidade e transformados em raiz quadrada de $\mathrm{x}+0,5$. A porcentagem de eficiência $(\% \mathrm{EF})$ dos inseticidas foi calculada pela fórmula de Abbott (1925).

Tabela 1. Ingrediente ativo, dose, grupo químico e modo de ação de inseticidas utilizados no controle de $C$. includens na cultura da soja (Experimento 1 e 2). Chapadão do Sul, MS.

\begin{tabular}{lccc}
\hline & & Experimento 1 & Modo de ação \\
\hline \multicolumn{1}{c}{ Ingrediente ativo } & Dose $\left(\mathrm{g}\right.$ i.a. ha $\left.{ }^{-1}\right)$ & Grupo Químico & - \\
\hline Testemunha & - & - & IEA + ISQ \\
Profenofós + Lufenuron & $15+150$ & ORG + BENZ & IEA \\
Metomil & 172 & CARB & ISQ \\
Teflubenzuron & 12 & BENZ & IEA \\
Thiodicarb & 160 & CARB & IEA \\
Clorpirifós & 384 & ORG & IEA + MCS \\
Fenitrotion + Esfenvalerate & $16+320$ & (ORG + PIR) & Modo de ação \\
\hline \multicolumn{1}{c}{ Ingrediente ativo } & Experimento 2 & - \\
\hline Testemunha & Dose $\left(\mathrm{g}\right.$ i.a. ha $\left.{ }^{-1}\right)$ & Grupo Químico & ISQ \\
Teflubenzuron & - & - & IEA \\
Metomil & 12 & BENZ & IEA / ISQ \\
Profenofos+ Lufenuron & 129 & CARB & ISQ / MCS \\
Flubendiamid & $15+150$ & ORG + BENZ & ISQ / MCS \\
Flubendiamid & 12 & DAF & DAF \\
\hline ORG Orga
\end{tabular}

ORG - Organofosforado; BENZ - Benzoiluréia; CARB - Carbamato; PIR - piretróide; DAF - Diamida do ácido ftálico; IEA- Inibidor da enzima acetilcolinesterase; ISQ- Inibidor da síntese da quitina; MCS- Modulador dos canais de sódio $\left(\mathrm{Na}^{+}\right)$. 


\section{Resultados e Discussão}

Aos 2, 14 e 21 dias após a aplicação (experimento 1) nenhum inseticida, nas doses testadas, diferiu da testemunha no número de lagartas pequenas (Tabela 2). Aos quatro dias os inseticidas fenitrotion + esfenvarelate diferiram testemunha, porém com eficiência não satisfatória de controle da praga $(75 \%$ de eficiência). No sétimo dia de avaliação os inseticidas metomil, tiodicarb, clorpirifós e fenitrotion + esfenvalerate apresentaram eficiências satisfatórias (81; $85 ; 81$ e $100 \%$, respectivamente).

Um inseticida pode ser considerado eficiente quando alcança, no mínimo, $80 \%$ de eficiência no controle de uma praga (TOMQUELSKI; MARTINS, 2007). Apesar dos carbamatos serem considerados eficientes no controle de lagartas da família Noctuidae (GALLO et al., 2002), no presente trabalho, thiodicarb e metomil foram eficientes no controle de lagartas pequenas somente no sétimo dia após a aplicação (Tabela 2). Resultados diferentes foram observados por Tomquelski \& Martins (2007) que estudando os carbamatos thiodicarb e metomil, verificaram que os mesmos foram eficientes três dias após a aplicação no controle de Spodoptera frugiperda em milho na região dos chapadões. Possivelmente, após o sétimo dia da aplicação, o residual dos inseticidas diminuiu e não proporcionou um controle satisfatório de $C$. includens.
Em relação ao controle de lagartas grandes, no sétimo dia após a aplicação profenofós + lufenuron, metomil e fenitrotion + esfenvalerate diferiram da testemunha no número de lagartas grandes, apresentando 7, 8 e 9 lagartas, respectivamente, enquanto a testemunha (sem aplicação) apresentou 37. $\mathrm{O}$ inseticida profenofos + lufenuron foi o mais eficiente $(81 \%)$ oferecendo resultado satisfatório no controle de C. includens (Tabela 2).

No décimo quarto e vigésimo primeiro dia após a aplicação não ocorreu resultados satisfatórios de controle. Nesse período, o tratamento com profenofos + lufenuron novamente apresentou maior eficiência no controle, porém na porcentagem de $52 \%$ somente aos 14 DAA (Tabela 2).

As benzoiluréias estudadas (lufenuron e teflubenzuron) não foram eficientes no controle de $C$. includens, aos 2, 4, 7, 14 e 21 DAA (Tabela 2). Estes resultados sugerem que existem diferenças na suscetibilidade de lagartas da família Noctuidae aos inseticidas pertencentes ao grupo das benzoiluréias, como reportado por Silva et al. (2003). Estes autores estudando o controle de Anticarsia gemmatalis, concluíram que lufenuron $\left(7,5\right.$ g i.a. ha $\left.{ }^{-1}\right)$, diflubenzuron $\left(15 \mathrm{~g}\right.$ i.a. $\left.\mathrm{ha}^{-1}\right)$ e teflubenzuron $(7,5 \mathrm{~g}$ i.a. $\mathrm{ha}^{-1}$ ) foram eficientes em avaliações realizadas aos 4, 7 e 10 dias após a aplicação.

Tabela 2. Efeito de inseticidas no controle de $C$. includens na cultura da soja. Número total (NT) de lagartas pequenas, grandes e porcentagem de eficiência (\%E) aos 0 (prévia), 2, 4, 7, 14 e 21 dias após a aplicação (DAA) (Experimento 1).

\begin{tabular}{|c|c|c|c|c|c|c|c|c|c|c|c|c|}
\hline \multicolumn{13}{|l|}{ Lagartas pequenas } \\
\hline \multirow{2}{*}{ Tratamentos } & \multirow{2}{*}{$\begin{array}{c}\text { Dose } \\
\left(\mathrm{g} \text { i.a. } \text { ha }^{-1}\right)\end{array}$} & \multicolumn{3}{|c|}{ Prévia 2 DAA } & \multicolumn{2}{|c|}{$4 \mathrm{DAA}$} & \multicolumn{2}{|c|}{$7 \mathrm{DAA}$} & \multicolumn{2}{|l|}{14 DAA } & \multicolumn{2}{|c|}{$21 \mathrm{DAA}$} \\
\hline & & NT & NT & $\% \mathrm{E}$ & NT & $\% \mathrm{E}$ & NT & $\% \mathrm{E}$ & NT & $\% \mathrm{E}$ & NT & $\% \mathrm{E}$ \\
\hline Testemunha & - & $101 \mathrm{a}$ & $80 \mathrm{a}$ & - & $32 \mathrm{a}$ & - & $26 a$ & - & $13 \mathrm{a}$ & - & $19 \mathrm{a}$ & - \\
\hline Profenofós+Lufenuron & $15+150$ & $94 \mathrm{a}$ & $62 \mathrm{a}$ & 22 & $10 \mathrm{ab}$ & 69 & $6 \mathrm{~b}$ & 77 & $29 \mathrm{a}$ & - & $25 \mathrm{a}$ & - \\
\hline Metomil & 172 & $128 \mathrm{a}$ & $75 \mathrm{a}$ & 6 & $17 \mathrm{ab}$ & 47 & $5 \mathrm{~b}$ & 81 & $17 \mathrm{a}$ & - & $25 \mathrm{a}$ & - \\
\hline Teflubenzuron & 12 & $111 \mathrm{a}$ & $70 \mathrm{a}$ & 12 & $11 \mathrm{ab}$ & 66 & $8 \mathrm{ab}$ & 69 & $16 \mathrm{a}$ & - & $12 \mathrm{a}$ & 37 \\
\hline Tiodicarb & 160 & $121 \mathrm{a}$ & $70 \mathrm{a}$ & 12 & $12 a b$ & 62 & $4 \mathrm{~b}$ & 85 & $12 \mathrm{a}$ & 8 & $18 \mathrm{a}$ & 5 \\
\hline Clorpirifós & 384 & $108 \mathrm{a}$ & $76 \mathrm{a}$ & 5 & $10 a b$ & 69 & $5 \mathrm{~b}$ & 81 & $10 \mathrm{a}$ & 23 & $22 \mathrm{a}$ & - \\
\hline Fenitrotion+Esfenvarelate & $16+320$ & $123 \mathrm{a}$ & $64 \mathrm{a}$ & 20 & $8 \mathrm{~b}$ & 75 & $0 \mathrm{~b}$ & 100 & $8 \mathrm{a}$ & 38 & $35 \mathrm{a}$ & - \\
\hline $\mathrm{CV}(\%)$ & & 22,1 & &, 4 & & & & 8,4 & & 4,3 & & 28,0 \\
\hline \multicolumn{13}{|l|}{ Lagartas Grandes } \\
\hline \multirow{2}{*}{ Tratamentos } & \multirow{2}{*}{$\begin{array}{c}\text { Dose } \\
\left(\mathrm{g} \text { i.a. } \mathrm{ha}^{-1}\right)\end{array}$} & Prévia & \multicolumn{2}{|c|}{2 DAA } & \multicolumn{2}{|c|}{$4 \mathrm{DAA}$} & \multicolumn{2}{|c|}{$7 \mathrm{DAA}$} & \multicolumn{2}{|l|}{$14 \mathrm{DAA}$} & \multicolumn{2}{|c|}{$21 \mathrm{DAA}$} \\
\hline & & NT & NT & $\% \mathrm{E}$ & NT & $\% \mathrm{E}$ & NT & $\% \mathrm{E}$ & NT & $\% \mathrm{E}$ & NT & $\% \mathrm{E}$ \\
\hline Testemunha & - & $68 \mathrm{a}$ & $69 \mathrm{a}$ & - & $31 \mathrm{ab}$ & - & $37 \mathrm{a}$ & - & $23 \mathrm{bc}$ & - & $1 \mathrm{a}$ & - \\
\hline profenofós+Lufenuron & $15+150$ & $51 \mathrm{a}$ & $44 \mathrm{a}$ & 36 & $13 \mathrm{~b}$ & 58 & $7 \mathrm{~b}$ & 81 & $11 \mathrm{~d}$ & 52 & $8 \mathrm{a}$ & - \\
\hline Metomil & 172 & $79 \mathrm{a}$ & $47 \mathrm{a}$ & 32 & $14 \mathrm{~b}$ & 55 & $8 \mathrm{~b}$ & 78 & $38 \mathrm{a}$ & - & $12 \mathrm{a}$ & - \\
\hline Teflubenzuron & 12 & $60 \mathrm{a}$ & $59 \mathrm{a}$ & 15 & $37 \mathrm{a}$ & - & $17 \mathrm{ab}$ & 54 & $39 \mathrm{a}$ & - & $3 \mathrm{a}$ & - \\
\hline Thiodicarb & 160 & $79 \mathrm{a}$ & $44 \mathrm{a}$ & 36 & $26 \mathrm{ab}$ & 16 & $30 \mathrm{a}$ & 19 & $18 \mathrm{~cd}$ & 22 & $6 \mathrm{a}$ & - \\
\hline Clorpirifós & 384 & $65 \mathrm{a}$ & $39 \mathrm{a}$ & 43 & $15 \mathrm{ab}$ & 52 & $19 a b$ & 49 & $15 \mathrm{~cd}$ & 35 & $2 \mathrm{a}$ & - \\
\hline Fenitrotion+Esfenvarelate & $16+320$ & $86 \mathrm{a}$ & $48 \mathrm{a}$ & 30 & $14 \mathrm{~b}$ & 55 & $9 \mathrm{~b}$ & 76 & $33 \mathrm{ab}$ & - & $2 \mathrm{a}$ & - \\
\hline $\mathrm{CV}(\%)$ & & 15,6 & & 6 & & & & 1,5 & & 0,9 & & 46,9 \\
\hline
\end{tabular}


As benzoiluréias inibem a formação da quitina sintetase a partir do seu zimógeno, pela interferência em alguma protease responsável pela ativação da quitina sintetase. Assim, larvas ou lagartas tratadas com estes inseticidas não podem libertar-se de sua exocutícula, por não conseguirem secretar endocutícula nova (RETNAKARAN et al., 1985; REYNOLDS, 1987).

No segundo experimento (Tabela 3), no segundo dia após a aplicação todos os tratamentos diferiram da testemunha em relação ao número de lagartas pequenas. Dentre os produtos testados, metomil, profenofos + lufenuron e flubendiamid (dose de 14,4 g i.a $\mathrm{ha}^{-1}$ ) foram os que apresentaram eficiência satisfatória com porcentagens acima de $80 \%$, ficando os outros produtos muito perto de atingirem esse valor; teflubenzuron com $71 \%$ e flubendiamide (dose de $12 \mathrm{~g}$ i.a ha $^{-1}$ ) com $77 \%$.

Aos 4 DAA de metomil, na dose de $129 \mathrm{~g}^{\text {i.a ha }}{ }^{-1}$, foi observada a presença de 2 lagartas pequenas, diferindo da testemunha que apresentou 29 (Tabela 3). Resultados semelhantes também foram encontrados por Tomquelski \& Martins (2006) na aplicação de 215 g i.a. ha $^{-1}$ de metomil para o controle de lagarta falsamedideira, comprovando a ação de choque do produto.

Os demais tratamentos não diferiram da testemunha e também do tratamento com metomil. A partir dos 7 DAA nenhum tratamento apresentou eficiência satisfatória $(\geq 80 \%)$. Aos 14 DAA, teflubenzuron, profenofos + lufenuron e flubendiamid (dose de 14,4 g i.a ha $^{-1}$ ) diferiram da testemunha, sendo que dois dos tratamentos citados (profenofos + lufenuron $\mathrm{e}$ flubendiamid) apresentaram maior eficiência (64\%) (Tabela 3).

Aos 2 DAA todos os tratamentos diferiram da testemunha para o controle de lagartas grandes, sendo o inseticida flubendiamid o mais eficiente (77\%) (Tabela 3). Aos quatro dias após a aplicação verifica-se que os tratamentos com teflubenzuron e Flubendiamid apresentaram eficiência acima de $80 \%$ de C. includens.

Os resultados observados aos 7 DAA evidenciam que os inseticidas profenofós + lufenuron e flubendiamid diferiram da testemunha, com eficiências de $74 \%$ e $84 \%$ respectivamente (Tabela 3). Estes inseticidas se sobressaíram devido aos seus modos de ação: inibidor da enzima acetilcolinesteraze + inibidor da síntese de quitina (profenofós + lufenuron) e modulador dos canais $\mathrm{Ca}^{+}$(flubendiamid), pois são reguladores hormonais do metabolismo de insetos, agindo algumas horas ou dias após as suas aplicações (GALLO et al., 2002).

Aos 14 DAA verificou-se que o tratamento com profenofós + lufenuron apresentou uma maior eficiência em relação aos demais tratamentos alcançando $71 \%$ do controle das lagartas (Tabela 2). A maior eficiência do inseticida profenofós + lufenuron observado no presente estudo pode estar associado à presença de dois modos de ação (organofosforado e benzoiluréia), apresentando ação de choque (profenofós) e somada à ação residual do regulador de crescimento (lufenuron). Inseticidas com apenas um mecanismo de ação (piretróides) apresentam acentuada ação de choque, porém, pequeno efeito residual (BORTOLINI; LECH, 2004; GUEDES et al., 2012).

Tabela 3. Efeito de inseticidas no controle de $C$. includens na cultura da soja. Número total (NT) de lagartas pequenas, grandes e percentagem de eficiência (\%E) aos 0 (prévia), 2, 4, 7, 14 DAA (Experimento 2).

\begin{tabular}{|c|c|c|c|c|c|c|c|c|c|c|}
\hline \multicolumn{11}{|l|}{ Lagartas Pequenas } \\
\hline \multirow{2}{*}{ Tratamentos } & \multirow{2}{*}{$\begin{array}{l}\text { Dose } \\
\left(\mathrm{g} \text { i.a. } \text { ha }^{-1}\right)\end{array}$} & \multirow{2}{*}{$\begin{array}{l}\text { Prévia } \\
\text { NT }\end{array}$} & \multicolumn{2}{|c|}{2 DAA } & \multicolumn{2}{|c|}{4 DAA } & \multicolumn{2}{|c|}{$7 \mathrm{DAA}$} & \multicolumn{2}{|c|}{14 DAA } \\
\hline & & & NT & $\% \mathrm{E}$ & NT & $\% \mathrm{E}$ & NT & $\% \mathrm{E}$ & NT & $\% \mathrm{E}$ \\
\hline Testemunha & - & $75 \mathrm{ab}$ & $44 \mathrm{a}$ & - & $29 \mathrm{a}$ & - & $34 \mathrm{a}$ & - & $14 \mathrm{a}$ & - \\
\hline Teflubenzuron & 12 & $65 \mathrm{ab}$ & $13 \mathrm{~b}$ & 71,0 & $11 \mathrm{ab}$ & 62 & $7 \mathrm{~b}$ & 79 & $7 \mathrm{~b}$ & 50 \\
\hline Metomil & 129 & $87 \mathrm{a}$ & $7 \mathrm{~b}$ & 84,0 & $2 \mathrm{~b}$ & 93 & $9 \mathrm{~b}$ & 74 & $9 \mathrm{ab}$ & 36 \\
\hline Profe+lufenuron & $15+150$ & $82 \mathrm{ab}$ & $7 \mathrm{~b}$ & 84,0 & $11 \mathrm{ab}$ & 62 & $7 \mathrm{~b}$ & 79 & $5 \mathrm{~b}$ & 64 \\
\hline Flubendiamid & 12 & $57 \mathrm{~b}$ & $10 \mathrm{~b}$ & 77,0 & $12 a b$ & 59 & $8 \mathrm{~b}$ & 77 & $8 \mathrm{ab}$ & 43 \\
\hline Flubendiamid & 14,4 & $74 \mathrm{ab}$ & $6 \mathrm{~b}$ & 86,0 & $8 \mathrm{ab}$ & 72 & $9 \mathrm{~b}$ & 74 & $5 \mathrm{~b}$ & 64 \\
\hline $\mathrm{CV}(\%)$ & & 8,29 & & 1 & & & & 2 & & 3,1 \\
\hline \multicolumn{11}{|l|}{ Lagartas Grandes } \\
\hline \multirow{2}{*}{ Tratamentos } & \multirow{2}{*}{$\begin{array}{l}\text { Dose } \\
\left(\mathrm{g} \text { i.a. } \mathrm{ha}^{-1}\right)\end{array}$} & Prévia & \multicolumn{2}{|c|}{2 DAA } & \multicolumn{2}{|c|}{4 DAA } & \multicolumn{2}{|c|}{7 DAA } & \multicolumn{2}{|c|}{14 DAA } \\
\hline & & NT & NT & $\% \mathrm{E}$ & NT & $\% \mathrm{E}$ & NT & $\% \mathrm{E}$ & NT & $\% \mathrm{E}$ \\
\hline Testemunha & - & $56 \mathrm{ab}$ & $43 \mathrm{a}$ & - & $42 \mathrm{a}$ & - & $31 \mathrm{a}$ & - & $17 \mathrm{a}$ & - \\
\hline Teflubenzuron & 12 & $49 \mathrm{~b}$ & $12 \mathrm{~b}$ & 72 & $7 \mathrm{~b}$ & 83 & $12 \mathrm{ab}$ & 61 & $7 \mathrm{~b}$ & 59 \\
\hline Metomil & 129 & $95 \mathrm{a}$ & $11 \mathrm{~b}$ & 74 & $11 \mathrm{~b}$ & 74 & $11 \mathrm{ab}$ & 65 & $6 \mathrm{~b}$ & 65 \\
\hline Profe+lufenuron & $15+150$ & $67 \mathrm{ab}$ & $11 \mathrm{~b}$ & 74 & $9 \mathrm{~b}$ & 79 & $8 \mathrm{~b}$ & 74 & $5 \mathrm{~b}$ & 71 \\
\hline Flubendiamid & 12 & $54 \mathrm{ab}$ & $12 \mathrm{~b}$ & 72 & $14 \mathrm{ab}$ & 67 & $16 \mathrm{ab}$ & 48 & $8 \mathrm{ab}$ & 53 \\
\hline Flubendiamid & 14,4 & $55 \mathrm{ab}$ & $10 \mathrm{~b}$ & 77 & $7 \mathrm{~b}$ & 83 & $5 \mathrm{~b}$ & 84 & $6 \mathrm{~b}$ & 65 \\
\hline $\mathrm{CV}(\%)$ & & 13,1 & \multicolumn{2}{|c|}{18,2} & \multicolumn{2}{|c|}{35,0} & \multicolumn{2}{|c|}{28,7} & \multicolumn{2}{|c|}{16,6} \\
\hline
\end{tabular}




\section{Conclusões}

Os inseticidas Fenitrotion+Esfenvarelate, Metomil, Tiodicarb, e Clorpirifós são os mais eficientes para o controle de lagartas pequenas de falsa-medideira Chrysodeixis includens (Lepidoptera: Noctuidae). Para as lagartas grandes os inseticidas mais eficientes são Flubendiamide e Profenofós + Lufenuron.

\section{Referências Bibliográficas}

ABBOTT, W. S. A method of computing the effectiveness of an insecticide. Journal of Economic Entomology, Lanham, v. 18, n. 1 , p. $265-267,1925$.

BORTOLINI, C. G.; LECH, A. R. M. Avaliação da eficiência de inseticidas no controle de lagartas das maçãs do algodoeiro e seletividade a inimigos naturais. Lucas do Rio Verde-MT: Fundação de Apoio à Pesquisa e Desenvolvimento Integrado de Rio Verde: resultados de pesquisa, Lucas do Rio Verde, 2004. 17p.

CONAB. COMPANHIA NACIONAL DE ABASTECIMENTO. Acompanhamento da safra Brasileira grãos, Safra 2014/15 - Décimo Levantamento. Brasilia-DF: 2015. Disponível em: http://www.conab.gov.br. Acesso em: 15 de junho de 2015.

DEGRANDE, P. E.; VIVAN, L. M. Pragas da Soja. Tecnologia e produção: soja e milho 2008/2009. MaracajuMS: FUNDAÇÃO MS, 2008. p. 73- 108.

EMBRAPA. EMPRESA BRASILEIRA DE PESQUISA AGROPECUÁRIA. Tecnologias de produção de soja região central do Brasil - 2009 e 2010. Londrina-PR: Embrapa Soja: Embrapa Cerrados: Embrapa Agropecuária Oeste, 2008.

GALLO, D.; NAKANO, O.; SILVEIRA NETO, S.; CARVALHO, R. P. L.; BAPTISTA, G. C.; BERTI FILHO, E.; PARRA, J. R. P.; ZUCCHI, R. A.; ALVES, S. B.; VENDRAMIM, J. D.; MARCHINI, L. C.; LOPES, J. R. S.; OMOTO, C. Entomologia Agrícola. Piracicaba-SP: FEALQ, 2002. 920p.

GUEDES, J. V. C.; FIORIN, R. A.; STURMER, G. R.; DAL PRA, E.; PERINI, C. R.; BIGOLIN, M. Sistemas de aplicação e inseticidas no controle de Anticarsia gemmatalis na soja. Revista Brasileira de Engenharia Agrícola e Ambiental, Campina Grande-PB, v. 16, n. 8, p. 910-914, 2012.

HANNIG. G. T.; ZEIGLER, M.; MARCON, P. G. Feeding cessation effects of chlorantraniliprole, a new anthranilic diamide insecticide, in comparison with several insecticides in distinct chemical classes and mode-of-action groups. Pest Management Science, Sussex, v. 65, n. 9, p. 969-974, 2009.
MARTINS, G. L. M.; TOSCANO, L.C.; TOMQUELSKI, G. MARUYAMA, W.I. Inseticidas no controle de Anticarsia gemmatalis (Lepidoptera: Noctuidae) e impacto sobre aranhas predadoras em soja. Revista Brasileira de Ciências Agrárias, Recife-PE, v. 4, n.2, p. 128-132, 2009.

MARTINS, G. L. M.; TOSCANO, L.C.; MARUYAMA, W.I.; TOMQUELSKI, G. Seletividade de inseticidas sobre predadores entomófagos na cultura da soja. Cultura Agronômica, Ilha Solteira-SP, v. 22, n. 2, p. 155-161, 2013.

MARTINS, G. L. M.; TOMQUELSKI, G.; PAPA, G. Aplicação de acybenzolar-s-methyl em algodoeiro para controle de Aphis gossypii (Glover) e Ramularia areola (Atkinson). Revista de Agricultura Neotropical, Cassilândia-MS, v. 2, n.1, p. 53-59, 2015.

RETNAKARAN, A.; GRANETT, J.; ENNIS, T. Insect growth regulators. In: KERKUT, G. A.; GILBERT, L. I. Comprehensive insect physiology biochemistry and pharmacology. New York: Pergamon, 1985. Cap. 12, p. 529-601.

REYNOLDS, S. E. The cuticule, growth regulators and moulting in insects: the essential background to the action of acylurea insecticides. Pesticide Science, Chichester, v. 20, n. 2, p. 131-146, 1987.

SILVA, M. T. B.; COSTA, E. C.; BOSS, A. Controle de Anticarsia gemmatalis Huebner (Lepidoptera: Noctuidae) com reguladores de crescimento de insetos. Ciência Rural, Santa Maria-RS, v. 33, n. 4, p. 601-605, 2003.

SOSA-GÓMEZ, D. R. Essa lagarta gosta de soja. Cultivar Grandes Culturas, Pelotas-RS, v.2, n.12, p.40-42, 2000

TOMQUELSKI, G. V.; MARTINS, G. L. M. Eficiência de inseticidas sobre Spodoptera frugiperda (J. E. Smith, 1797) (Lepidoptera: Noctuidae) em milho na região dos Chapadões. Revista Brasileira de Milho e Sorgo, Sete Lagoas-MG, v. 6 , n. 1, p. 26-39, 2007.

TOMQUELSKI, G. V.; MARTINS, G. M. Amostragem de pragas da soja. Pesquisa, Tecnologia e Produtividade, Chapadão do Sul-MS, v. 1, n. 6, p. 44-46, 2012.

TOMQUELSKI, G.V.; MARTINS, G. L. M.; DIAS, T. S. Características e manejo de pragas da cultura da soja. Pesquisa, Tecnologia e Produtividade, Chapadão do SulMS, v. 2, n. 9, p. 61-82, 2015.

UNIVERSIDADE FEDERAL DE VIÇOSA - UFV. SAEG. Sistemas de análises estatísticas e genéticas. Versão 8.0. Viçosa, MG, 2001. 301 p. (Manual do usuário). 